\title{
O Ensino de Geriatria e Gerontologia na Graduação Médica
}

\section{PALAVRAS-CHAVE \\ - Currículo; \\ - Educação Médica; \\ - Geriatria.}

\section{Teaching of Geriatrics and Gerontology at Medical School}

Vitor Jorge Woytuski Brasil ${ }^{I}$

Nildo Alves BatistaII

\begin{abstract}
RESUMO
Este ensaio evidencia aspectos de relevância para o ensino de Geriatria e Gerontologia nas escolas médicas brasileiras, balizados na literatura atual sobre a temática e no diálogo com docentes de Geriatria. Vislumbramos a inserção acadêmica da Geriatria e Gerontologia como uma aproximação gradual das escolas do processo de envelhecimento e suas consequências médico-sociais. Para que isto ocorra, enfatizamos a utilização de ações verdadeiramente interdisciplinares que perpassem de forma longitudinal todos os anos do curso médico e integrem as demais especialidades. A ampliação e diversificação dos cenários formais de aprendizagem, além da preocupação com a capacitação e qualificação docente nesta área foram identificadas como pilares indispensáveis à formação de profissionais que possam atender de forma adequada às necessidades de saúde da população idosa nas próximas décadas.
\end{abstract}

\begin{abstract}
This study highlights relevant aspects for teaching Geriatrics and Gerontology at Brazilian medical schools in accordance with current literature on the subject and on dialogues with Geriatrics teachers. We focus on academic insertion of geriatrics and gerontology as a form of gradually bringing schools more in touch with the aging process and its social-medical consequences. We therefore emphasize the use of real interdisciplinary actions that cross through all the years of the medical course and integrate it with other specialties. The expansion and diversification of formal learning scenarios, as well as the concern with teacher training and qualification in this area, have been identified as essential pillars for preparing professionals who can adequately meet the health needs of the elderly population in the coming decades.
\end{abstract}

Recebido em: 01/05/2013

Reencaminhado em: 08/08/2014

Aprovado em: 31/08/2014 


\section{INTRODUÇÃO}

Nosso país vem se destacando como uma potência econômica emergente na conjuntura globalizada atual. No entanto, ainda temos problemas clássicos, inerentes à maioria dos países pobres, como altos índices de analfabetismo, falta de saneamento básico, déficit habitacional, tripla carga de doenças, transporte público oneroso e pouco eficiente ${ }^{1,2}$.

Com todas estas variáveis, quando se analisam índices populacionais e epidemiológicos do nosso país continental, os dados são inconstantes e pouco fidedignos, gerando conflitos nas tomadas de decisão e na priorização das demandas do planejamento estratégico em saúde $e^{2,3}$.

Um dos índices populacionais globais mais debatidos se refere à transição demográfica, observada há mais de um século, que evidencia um progressivo envelhecimento da população mundial. Este índice ocorre de forma gradual e linear nos últimos dois séculos em países ricos². Em países emergentes, como o Brasil, em menos de cem anos esta transição se apresenta de forma exponencial, acelerada e desordenada. Infelizmente, o envelhecimento da população brasileira é vislumbrado de forma dicotômica: ora é um fator de progresso e avanço socioeconômico, ora como mais um "problema" a ser priorizado e solucionado ${ }^{2-5}$.

O envelhecimento humano é um processo que dura toda a vida, por meio de mudanças no estado biológico, psicológico, social e espiritual, aparecendo já na fase intrauterina e continuando ao longo de toda a existência ${ }^{1}$. Pelo ciclo vital, a velhice é a penúltima etapa, podendo ser a de maior duração. $\mathrm{O}$ indivíduo que se encontra nessa fase da vida é denominado idoso, sendo reconhecido socialmente por uma mensuração cronológica - indivíduos com mais de 60 anos nos países em desenvolvimento, e maiores de 65 anos nos países desenvolvidos ${ }^{6}$.

Atualmente, o relógio biológico da espécie humana atinge 90-95 anos, com ajuste gradativo nas próximas décadas para até os limites de 130 anos, com a finalização desta fase com a morte. As mudanças inerentes à velhice que ocorrem no comportamento, na habilidade intelectual e na capacidade física afetam diretamente a interação do indivíduo com o meio ambiente, e, de acordo com a cultura, o idoso constitui um elemento fundamental para o (des)equilíbrio de determinada sociedade ${ }^{6-8}$.

Como tema de estudo, a velhice possui teorias e reflexões que remontam à idade antiga. Provavelmente, o primeiro manuscrito da sociedade ocidental remete ao filósofo Cicero (10642 a.C.), da Roma antiga, que, em seu manuscrito Senectude, aborda os aspectos relevantes da heterogeneidade da velhice e orientações específicas para condições mórbidas de alta prevalência nesta fase da vida ${ }^{9}$
Os fundamentos das ciências dedicadas ao envelhecimento humano tiveram seu embasamento teórico moderno no início do século XX. Em 1903, Elie Metchikoff defendeu a criação de uma nova especialidade, a Gerontologia, criada a partir da fusão das palavras géron (velho) e logia (estudo). Géron é uma expressão de origem grega, que remete ao conselho de 28 membros da Gerúsia ou Senado, em Esparta. Os privilegiados deste seleto grupo eram denominados gerontes e deveriam ter no mínimo 60 anos? $^{9}$.

A Gerontologia, por definição, é a área do conhecimento científico voltada para o estudo do envelhecimento em sua perspectiva mais ampla, que leva em consideração não somente os aspectos clínicos e biológicos, mas também as condições psicológicas, sociais, econômicas, históricas e suas invariáveis interações ${ }^{6,9}$.

Em convergência com os princípios da Gerontologia, o doutor Ignatz L. Nascher, médico vienense radicado nos Estados Unidos, estabeleceu em 1909 o conceito de Geriatria, como o ramo da ciência médica voltado à promoção da saúde e ao tratamento de doenças e incapacidades na velhice ${ }^{9}$. O estabelecimento formal das ciências do envelhecimento no Brasil ocorreu somente após cinco décadas, em 1961, quando foi fundada a Sociedade Brasileira de Geriatria. Após sete anos, a Sociedade incorporou profissionais de outras formações, transformando-se na Sociedade Brasileira de Geriatria e Gerontologia (SBGG), afiliada à International Association of Gerontology (IAG) e à Associação Médica Brasileira (AMB) .

A difusão deficiente do conhecimento geriátrico junto aos profissionais de saúde ainda na graduação tem contribuído decisivamente para as dificuldades na abordagem médica deste paciente ${ }^{10}$. Desta forma, o plano de ação internacional de Madri sobre o envelhecimento humano destacou como um de seus objetivos melhorar as informações e a capacitação dos profissionais de saúde e serviços sociais sobre as necessidades das pessoas idosas ${ }^{11}$, ressaltando urgência de ações de estímulo ao ensino de Geriatria na graduação, com enfoque estratégico nos países em desenvolvimento.

Porém, a Geriatria e a Gerontologia estão incorporadas em menos da metade dos projetos pedagógicos de graduação médica das escolas brasileiras, com maior ênfase percentual nas graduações médicas das regiões Sul e Sudeste. Isto demonstra extrema divergência para o adequado desenvolvimento de conhecimentos, habilidades e primordialmente atitudes do médico brasileiro no atendimento desta população ${ }^{12}$.

Diante disso, vamos discutir itens considerados relevantes para o ensino da Geriatria e Gerontologia na graduação médica, adequados ao contexto do nosso país, harmonizando-se com alguns princípios do Sistema Único de Saúde (SUS), como transversalidade, integralidade e universalidade ${ }^{3-10}$. 
A base do artigo dialoga com dados da literatura atual sobre a temática em questão e com concepções de docentes especialistas em Geriatria inseridos em escolas médicas da Regional Sul II da $\mathrm{Abem}^{13}$, todos com pelo menos dez anos de docência.

Esta discussão está balizada na dissertação de mestrado defendida e aprovada na Universidade Federal de São Paulo pelo autor principal. A tese foi desenvolvida segundo os procedimentos éticos previstos pelo Conep e aprovada pelo Comitê de Ética/Unifesp (Processo 1356/04) ${ }^{14}$.

\section{A INTERDISCIPLINARIEDADE: FUNDAMENTO BÁSICO}

Em sua gênese, a prática profissional com fundamentos gerontológicos é necessariamente interdisciplinar. O nível de excelência de organização e sistematização no atendimento ao paciente geriátrico é identificado com base na constituição de experiências interdisciplinares, sendo fundamental para a formação do profissional de saúde e indispensável no atendimento deste segmento populacional ${ }^{15,16}$.

Neste sentido, a implantação efetiva da interdisciplinaridade no ensino de Geriatria e Gerontologia na graduação é um ponto unânime entre os docentes.

"Eu acho que um bom ensino de Geriatria implica interdisciplinaridade $[. . .]^{\prime \prime}$

"[...] modelos interdisciplinares mais amplos do que simplesmente a gente se deter só na abordagem tradicional, que é importante, mas não é suficiente para o idoso".

"[...] a Geriatria e Gerontologia não podem ser dadas só pela Geriatria, é uma rede com várias áreas [...]"

Com base nesta premissa, em 2008, a American Geriatric Society (AGS) convidou especialistas de diversas áreas (Odontologia, Medicina, Enfermagem, Nutrição, Terapia Ocupacional, Farmácia, Terapia Ocupacional, Psicologia e Assistência Social) para constituir um grupo de trabalho em prol da saúde do idoso, o Partnership for Health in Aging (PTH). Este grupo evidencia pelo menos seis aspectos primordiais para o desenvolvimento de estratégias de ensino interdisciplinares: promoção e segurança da saúde; entrevista e instrumentos de avaliação do idoso; coordenação e planejamento da assistência; formação de equipe interdisciplinar; apoio ao cuidador; sistemas de saúde e benefícios ${ }^{15}$.

Nas escolas estadunidenses, as estratégias curriculares de 65\% delas incorporam como habilidades necessárias à atuação em saúde da pessoa idosa a participação em equipes interdisiciplinares ${ }^{17}$.

Muitas dessas instituições adotaram um projeto personalizado, denominado Geriatric Interdisciplinary Team Training (GITT), onde, desde 1997, promovem a capacitação gerontológica com fundamentos interdisciplinares. Este projeto é idealizado e estruturado pela fundação americana The John Hartford Foundation, que há quase cem anos desenvolve projetos estratégicos de pesquisa e educação na área do envelhecimento e saúde ${ }^{18}$

Na Columbia University, a interdisciplinaridade congrega diversos profissionais por meio do programa Columbia Cooperative Aging Program, que intercala conceitos, instrumentos e técnicas de assistência, caracterizando-se como uma conexão fundamental para a atenção do indivíduo idoso ${ }^{19}$.

A Academia Latinoamericana de Medicina del Adulto Mayor (Alma $)^{20}$ - entidade que tem apoio direto da Organização Pan-Americana de Saúde (Opas) e que desde o ano 2000 congrega exclusivamente docentes com inserção no ensino de Geriatria nas escolas médicas da América Latina e Caribe aponta como ponto crucial no ensino da graduação médica a existência de espaços formais para a Geriatria e Gerontologia e que estes espaços devem ter autonomia e autoridade suficientes para determinar seu programa de ensino, com uma integração constante com as outras especialidades e departamentos $^{21}$.

A universalidade da atenção é contemplada quando um dos docentes realça a importância de considerar este ensino de forma transversal no currículo, com a inserção em mais de um momento de formação do futuro médico.

\footnotetext{
"Mas você só vai conseguir estruturar um curso como este se tiver disponibilidade dentro do currículo. Então, eu acho que ele pode estar inserido em vários momentos".
}

Esta universalidade também pode estar conectada com outro aspecto de excelência para um adequado ensino médico, que a insere na perspectiva do curso de vida, com os aspectos de envelhecimento humano desde o início da vida intrauterina até a morte $e^{17,22,23}$

\section{A TRANSVERSALIDADE COMO MEIO DE EXCELÊNCIA}

Com uma visão ainda tímida para a construção estruturante dos aspectos referentes à totalidade do ciclo vital, porém ímpar no ensino da Geriatria na graduação médica brasileira, aliada a uma experiência pioneira de transversalidade curricular, a disciplina de Geriatria e Gerontologia da graduação 
médica da Universidade Federal de São Paulo conseguiu perpassar aspectos gerontogeriátricos por toda a graduação médica - desde os ciclos básicos, na primeira e segunda séries, profissionalizante, na terceira e quarta séries, até o internato, na quinta série -, ajustando linearmente o nível de complexidade na abordagem deste indivíduo ${ }^{24}$.

A abordagem em saúde relacionada à pessoa idosa ainda está muito centrada no atendimento médico, procurando explicar todas as queixas e sinais apresentados em um único diagnóstico, mas esta prática é totalmente inadequada quando aplicada à pessoa idosa ${ }^{25}$.

A integralidade na atenção à pessoa idosa está além das questões biológicas do processo saúde-doença no envelhecimento humano e mostra-se fundamental no processo de ensino do futuro médico:

\section{"[...] para discutir esta questão não biológica da saúde, a questão psíquica, a questão social, questão política, econômi- ca, a questão familiar, previdenciária, então acho que nós, ge- riatras, temos que passar na graduação estas dimensões não biológicas do processo de envelhecimento".}

Na discussão sobre a integralidade do cuidado, afirma-se a necessidade de aprimorar o acolhimento, entendido como um olhar humanizante perante toda a demanda pelas ações de saúde, considerando este vínculo em três dimensões: afetividade, relação terapêutica e continuidade ${ }^{26}$. Para os profissionais de saúde que atendem o idoso, resgatar o sentido do cuidado é estratégico:

\footnotetext{
"uma ação integral que tem significados e sentidos para se compreender a saúde como direito de ser. É tratar, respeitar, acolher. Atender o ser humano em seu sofrimento, em grande medida fruto de sua fragilidade social".
}

Sendo assim, é essencial uma avaliação abrangente do idoso, com enfoque nos aspectos físicos, psicológicos, sociais, culturais e econômicos, caracterizando uma avaliação global das suas condições ${ }^{6,27}$. No âmbito da saúde pública, esta avaliação teve sua sistematização com a implementação dos $\mathrm{Ca}$ dernos de Atenção Básica, cujo número 19 apresenta a sistemática mínima para uma avaliação direcionada e com abrangência humanizada, centrada na pessoa ${ }^{28}$.

Contudo, a aplicação destes preceitos não é nada contemporânea. Seus alicerces estão nos primórdios da medicina geriátrica moderna, quando, no início do século $X X$, no West Middlesex Hospital da Inglaterra, a Drª . Marjory Warren prontificou-se a avaliar e reabilitar pacientes crônicos que praticamente residiam no hospital, restabelecendo o convívio social de vários indivíduos idosos ${ }^{29}$.

Por meio da perpetuação das tendências verificadas pela dinâmica demográfica da população nacional, as projeções para as próximas duas décadas demonstram que os idosos poderão representar até $13 \%$ da população, chegando a mais de 30 milhões. Além disso, a proporcionalidade dos idosos "mais idosos", ou seja, maiores de 80 anos, também está aumentando, alterando a composição dentro do próprio grupo. Desta forma, embora a fecundidade ainda seja o principal fator do processo com relação à população idosa, é a longevidade que vem progressivamente definindo seus traços de evolução $0^{2,5,30}$.

\section{A ÚLTIMA FASE OUE NÃO É O FIM}

Assim, a convivência cada vez maior com o idoso muito idoso e a necessidade de maior motivação e preparo para os cuidados paliativos são desafios a superar na graduação do médico.

\begin{abstract}
"[...] convencer o acadêmico, quer ele queira ou não, vai chegar um momento em que ele vai precisar conhecer a medicina paliativa, o individuo doente num estado de irreversibilidade que vai viver por meses ou até anos com uma limitação grande, com grau de sofrimento grande, e que a gente não vai poder oferecer grandes ferramentas ou grandes técnicas que irão reverter isso; ao contrário, ele vai ter que conviver com algumas realidades e nós temos que oferecer para ele, dentro desta realidade, o mínimo de suporte para que ele minimize o sofrimento e mantenha a sua dignidade".
\end{abstract}

Há uma correlação direta entre os processos de transição demográfica e epidemiológica. Geralmente, o início da queda da mortalidade se concentra seletivamente entre as doenças infectocontagiosas, tendendo a beneficiar os grupos mais jovens da população ${ }^{1,4}$. Estes sobreviventes passam a conviver com fatores de risco para distúrbios crônico-degenerativos, e, à medida que aumenta a expectativa de vida, tornam-se mais frequentes as complicações destes distúrbios, que inevitavelmente implicam décadas de utilização dos serviços de saúde ${ }^{30}$. Como exemplos, destacam-se as sequelas de distúrbios cardiovasculares, fraturas decorrentes de quedas, amputações e cegueira provocadas pelo diabetes e a dependência determinada pelos processos de deterioração cognitiva, em que o preparo do futuro médico para esta nova realidade é essencial ${ }^{2,7,10-11}$.

\section{UM MUNDO DE TODAS AS IDADES}

A ampliação e a flexibilização de cenários de ensino-aprendizagem, rompendo com a ênfase hospitalocêntrica do ensino médico, são estratégias importantes para o ensino de Geriatria e Gerontologia na graduação médica. 
"[...] o ambiente hospitalocêntrico, como a gente chama, é muito bom para a Medicina Interna, mas para a Geriatria não é o ideal".

Neste sentido, a diversificação de locais de prática que possibilite ao estudante a vivência de diferentes cenários do cotidiano de um idoso possibilita ampliar o entendimento do campo da Geriatria e da Gerontologia ${ }^{31}$.

Múltiplos ambientes estimulam uma variabilidade de pacientes com diferentes estratos sociais, econômicos, de gênero e étnicos, aliados a diferentes experiências de diagnóstico e manejo de doenças geriátricas, prescrição de fármacos e estratégias de reabilitação segundo o local e o nível de complexidade em questão, além de estimularem maior interação com profissionais de saúde e até de outras áreas ${ }^{31,32}$.

Quase todas as escolas americanas (95\%) que possuem Geriatria em seu currículo adotam espaços diversificados para o seu ensino, prioritariamente em instituições de longa permanência, domicílio e ambulatórios na comunidade ${ }^{33}$. Observa-se a migração dos espaços de ensino da Geriatria para espaços educacionais não tradicionais, cuja facilitação se deve à implementação de novas tecnologias educacionais, a exemplo do Consortium of E-Learning in Geriatrics Instruction (Celgi), que utiliza inúmeras ferramentas virtuais ${ }^{34}$.

Para implantar um ensino mais relevante, a configuração das políticas relacionadas ao envelhecimento é fundamental, especialmente as políticas públicas de atenção ao idoso e as políticas de formação de recursos humanos para a Geriatria e Gerontologia.

"Eu acho que, em geral, em termos de política, política assistencial, [...] A saúde em relação a Ministério da Saúde, Ministério da Previdência, a instância federal ainda não está sensibilizada para a importância da atenção geriátrica".

As políticas públicas brasileiras integradas à Secretaria de Assistência Social do Ministério da Previdência e Assistência Social, em 1976, já decretavam a "Era do Envelhecimento: 1975-2025", embaladas pelas iniciativas dos organismos internacionais que foram sedimentados pelas projeções epidemiológicas e demográficas ${ }^{35}$.

O seminário "Estratégias de Política Social para o Idoso no Brasil" visou colher subsídios e sugestões para a adoção de um programa especialmente voltado ao benefício da população idosa do País. Esse evento deu margem à criação de dois espaços fundamentais para a formatação das políticas futuras. Integrando-se às orientações do Plano de Ação Internacional sobre Envelhecimento desenvolvido na Assembleia Mundial das Nações Unidas em 1982 em Viena e, sequencialmente, em 1985, com a elaboração de um programa sobre a saúde do idoso pela Opas, foi desenvolvido em 1994 o Programa Nacional do Idoso, voltado às políticas de atenção ao idoso em diversas áreas ${ }^{10,35}$.

Após estes marcos iniciais, foram implementadas inúmeras outras estratégias, que culminaram na criação da Política Nacional do Idoso em 1996 e na implementação da Política Nacional de Saúde do Idoso (PNSI) em 1999. Esta já apresentava como propósito basilar aspectos inerentes de prevenção, promoção, manutenção e recuperação da saúde, especialmente norteados pelas noções centralizadas na manutenção da capacidade funcional e não em doenças ${ }^{10-36}$.

A PNSI propõe que o desenvolvimento e a capacitação de recursos humanos constituam uma prioridade, perpassando todas as demais definidas nesta estratégia. Este é um mecanismo privilegiado de articulação intersetorial, sendo que o setor saúde pode dispor de pessoal em qualidade e quantidade adequadas, e cujo provimento seria de responsabilidade das três esferas de governo ${ }^{36}$.

A Lei n ${ }^{\circ} 8.080 / 90$, em seu Artigo 14, estabelece que a formação e a educação continuada configurem ações intersetoriais articuladas. Estabelece, ainda, a criação de comissão permanente de integração entre os serviços de saúde e as instituições de ensino profissional e superior, propondo prioridades, métodos e resultados ${ }^{37}$.

A 2 ${ }^{\mathrm{a}}$ Assembleia Mundial sobre o Envelhecimento, promovida pela ONU, destaca: a expansão da educação profissional em Gerontologia e Geriatria; a inclusão de treinamento multidisciplinar; a formação básica e especializada sobre a saúde do idoso; a promoção de programas de educação continuada em saúde, bem-estar e cuidado de pessoas idosas para profissionais da área da saúde, da assistência social e cuidadores; o estímulo ao aumento de estudantes na área e a promoção de programas de treinamento em Gerontologia e Geriatria, em especial nos países em desenvolvimento, mediante cooperação bilateral e multilateral internacional.

Todas estas prerrogativas são contempladas em lei, através do Estatuto do Idoso, e reforçadas por meio da reedição da Política de Saúde do Idoso, com a Portaria do Ministério da Saúde n⿳o 2.528 de 19 de outubro de 2006, agora acertadamente denominada Política Nacional de Saúde da Pessoa Idosa ${ }^{38,39}$.

\section{QUEM VAI CUIDAR DE NÓS?}

A todos estes aspectos soma-se o desafio de encontrar estratégias de capacitação docente para o Ensino da Geriatria e Gerontologia. 
"[...] hoje as universidades brasileiras têm muito pouco professor com formação para ministrar aulas de Geriatria e existem poucas vagas de residência e é a residência que irá formar o futuro docente".

De maneira ainda incipiente e sem padrões mínimos temáticos, ocorrem os cursos de extensão universitária e de pós-graduação lato sensu (Especialização) e stricto sensu (Mestrado e Doutorado), que deveriam ser responsáveis pela formação e capacitação de profissionais capazes de efetuar e planejar o atendimento da população idosa ${ }^{40}$.

Esta estrutura é amplamente incentivada em diversas sociedades ao redor do mundo. Nos Estados Unidos, a Gerontological Society of America possui, desde 1974, uma associação específica voltada aos estudos e avanços pedagógicos do ensino gerontogeriátrico, a Association for Gerontology in Higher Education (Aghe $)^{41}$. Tem como missão promover o avanço da educação em Gerontologia e Geriatria nas universidades, bem como a liderança e o apoio a educadores e estudantes nas instituições de ensino, e edita um periódico científico direcionado exclusivamente ao ensino de Geriatria e Gerontologia.

A Europe Union Medicine Geriatric Society possui o Special Interest Group (SIG) ${ }^{42}$, cujo enfoque principal é promover a qualidade da educação, formação e avaliação da medicina geriátrica europeia. Pessoalmente, destacamos os tradicionais centros franceses e ingleses.

Entendemos que, para o melhor exercício da docência em Geriatria e Gerontologia como área peculiar do conhecimento médico, é necessário um conhecimento mínimo na área.

Do ponto de vista acadêmico, a residência médica poderia ser o caminho inicial mais privilegiado. Porém, a priori, a residência em Geriatria no País, de forma semelhante à maioria das residências de outras especialidades, está configurada para a assistência e não para os aspectos mínimos da docência e da pesquisa, conforme estabelecido na Resolução do Conselho Nacional de Residência Médica (2003) ${ }^{43}$. E, mesmo assim, possuímos em todo o território nacional somente 35 programas de residência ${ }^{44}$

Neste contexto, muitos profissionais médicos descobrem-se professores recém-formados dos programas de residência médica e, em muitas situações, também identificam que os cursos de pós-graduação stricto sensu não asseguram proficiência para educar novos profissionais. Com isso, este novo profissional necessita de um programa de formação permanente para tornar-se efetivamente um professor de Medicina e em especial de Geriatria e Gerontologia ${ }^{45,46}$.

Enfim, o ensino de Geriatria e Gerontologia ainda é incipiente na maioria das escolas médicas brasileiras. Eliminar as barreiras para a sua adequação e sedimentação nos programas curriculares e definir um modelo de formação condizente com as peculiaridades da assistência ao idoso no País são metas a alcançar.

As demandas relacionadas à pessoa idosa não serão supridas em curto ou médio prazo, devendo ser observadas de forma permanente. Para isso, é primordial viabilizar a difusão da noção de envelhecimento humano como um processo natural de todos os seres humanos, e a mola propulsora para esta transformação, sem dúvida, se encontra nos espaços identificados como acadêmicos.

\section{REFERÊNCIAS}

1. Chaimowicz FA. Saúde dos idosos brasileiros às vésperas do século XXI: problemas, projeções e alternativas. Rev. Saúde Pública. 1997; 31(2):184-200.

2. Veras RP. Envelhecimento populacional contemporâneo: demandas, desafios e inovações. Rev. Saúde Pública. 2009; 43(3):548-54.

3. Brasil. Ministério da Saúde. Vigilância em Saúde. Saúde Brasil 2004: uma análise da situação de saúde. Brasília: Ministério da Saúde; 2004. p. 52-55.

4. Camarano AA. Envelhecimento da população brasileira: uma contribuição demográfica. In: Freitas EV, Py L, Nery AL, Cançado FAX, Gorzoni ML, Rocha SM, orgs. Tratado de Geriatria e Gerontologia. Rio de Janeiro: Guanabara Koogan; 2002. p. 58-71.

5. Veras RP. Envelhecimento populacional e as informações de saúde no PNAD: demandas e desafios contemporâneos. Cad. Saúde Pública. 2007; 23(10):2463-66.

6. Papaléo Netto M. O estudo da velhice no século XX: histórico, definição do campo e termos básicos. In: Freitas EV, Py L, Nery AL, Cançado FAX, Gorzoni ML, Rocha SM, orgs.Tratado de Geriatria e Gerontologia. Rio de Janeiro: Guanabara Koogan; 2002. p. 2-12.

7. Kalache A, Veras RP, Ramos LR. O envelhecimento da população mundial: um desafio novo. Rev. Saúde Pública. 1987; 21: 200-10.

8. World Health Organization. Active Ageing: a policy framework. Geneva: World Health Organization; 2002.

9. Morley JE. A brief history of geriatrics. Journal of Gerontology. 2004; 59(11): 1132-1152.

10. Brasil. Ministério da Saúde. Portaria n. ${ }^{\circ}$ 1.395, de 9 de dezembro de 1999. Diário Oficial [da] República Federativa do Brasil. Política Nacional de Saúde do Idoso. Brasília; 2001.

11. United Nations. The Second World Assembly on Ageing 2002(Espanha) The third meeting of the technical commmitee for the Second World Assembly on Ageing, 2002 
[capturado 01 jun 2014] Disponível em: http:/ / undesadspd.org/Portals/0/ageing/documents/Fulltext-E.pdf.

12. Pereira AMVB, Feliz MC, Schwanke CHA. Ensino de Geriatria nas faculdades de medicina brasileiras. Rev Geriatria \& Gerontologia. 2010;4(4):179-185.

13. Associação Brasileira de Educação Médica. 2006 [capturado 17 mar 2007]. Disponível em: www.abem-educmed.org. br/imagens/botoes/novos/escolas2.jpg

14. Brasil VJW. A Geriatria e Gerontologia nos currículos formais das escolas médicas do Paraná. São Paulo; 2007. Mestrado [Dissertação] - Centro de Desenvolvimento do Ensino Superior em Saúde (CEDESS), Universidade Federal de São Paulo.

15. Partnership for Health in Aging Workgroup on Multidisciplinary Competencies in Geriatrics. Multidisciplinary Competencies in the Care of Older Adults at the Completion of the Entry-level Health Professional Degree [capturado 20 jun. 2014]. Disponível em: http:/ / www.americangeriatrics. org/files/documents/pha/PHAMultidiscComps.pdf

16. Montagnini RM, Kaiser GC, Melanie AD, Carol G; Vyjeyanthi SP, Dee R, Sandra E.Sanchez-Reilly, Todd PS, Heather S, et al. Position statement on interdisciplinary team training in geriatrics: An essential component of quality health care for older adults. Journal of the American Geriatrics Society.. 2014;62;5 [capturado 20 jun. 2014]. Disponível em: http: / / www.americangeriatrics.org/files/documents / Full_IDT_Statement.pdf.

17. Eleazer GP, Doshi R, Wieland D, Boland R, Hirth VA. Geriatric content in medical school curricula: results of a national survey. J AM Geriatr Soc. 2005; 53:136-40.

18. Fulmer T, Hyer K, Flaherty E, Mezey M, Whitelaw N, Jacobs MO, Luchi R, Hansen JD, Evans DA, Cassel C, Kotthoff-Burrell E, Kane R, Pfeiffer E. Geriatric interdisciplinary team training program: Evaluation results. Journal of Aging and Health. 2005; 17(4):443-470.

19. Maurer SM, Costley AW, Miller, PA, McCabe S, Dubin S, Cheng $\mathrm{H}$, et al. The Columbia Cooperative Aging Program: An Interdisciplinary and Interdepartmental Approach to Geriatric Education for Medical Interns. Journal of the American Geriatrics Society. 2006; 54(3):520-526.

20. Cano C, Gutiérrez LM, Marín PP, Morales Martínez F, Peláez M, Mañaz L, et al. Propuesta de contenidos mínimos para los programas docentes de pregrado en Medicina Geriátrica en América Latina. Rev Panam Salud Pública. 2005; 17(5-6):429-37.

21. Morales Martinez, F. Educatión gerontológica formal. In: Anzola PE. La atencion de los ancianos: um deseño para los años noventa. Washington DC: OPAS; 1994.
22. American Geriatrics Society Education Committee and Policy Advisory Group. Education in Geriatric Medicine. Journal American Geriatrics Society. 2001; 49: 223-4.

23. World Health Organization and International Longevity Centre-UK. The Implications for Training of Embracing a Life Course Approach to Health. Geneva: World Health Organization; 2000.

24. Cendoroglo MS, Araujo LMQ, Pontes JR, Povineli BMS, Piovezan RD, Santos FC, et al. Ensino de Geriatria e Gerontologia na Graduação Médica - UNIFESP. In: Anais do $5^{\circ}$ Congresso Paulista de Educação Médica; 2006, Botucatu, Brasil

25. Veras RP. Em busca de uma assistência adequada à saúde do idoso: revisão da literatura e aplicação de um instrumento de detecção precoce e de previsibilidade de agravos. Cad. Saúde Pública. 2003; 19(3): 705-15.

26. Marins JJN, Rego S, Lampert JB, Araujo JGC, orgs. Educação médica em transformação: instrumentos para a construção de novas realidades. São Paulo: Hucitec; 2004.

27. Fitzgerald JT, Williams BC, Halter JB, Remington Tl, Foulk MA, Persky NW, Shay BR. Effects of a geriatrics interdisciplinary experience on learners' knowledge and attitudes. Gerontology \& Geriatrics Education 2006;26(3):17-28.

28. Brasil. Ministério da Saúde, Secretaria de Atenção à Saúde, Departamento de Atenção Básica. Envelhecimento e saúde da pessoa idosa. Brasília; 2006. 192 p. il. - [Série A. Normas e Manuais Técnicos][Cadernos de Atenção Básica, n. 19].

29. Matthews DA. Dr. Marjory Warren and the origin of british geriatrics. Journal American Geriatric Society. 1984; 32(4): 253-8.

30. Luis FVF, Priscila MA, Francisca VSS, Vanessa MB, Luzeni GS, Amanda CLM, et al. Epidemiologia do envelhecimento: dinamização, problemas e consequências. Revista Kairós Gerontologia. 2012; 15(2): 55-69.

31. Oates DJ, Norton LE, Russell ML, Chao SH, Hardt EJ, Brett $\mathrm{B}$, et al. Multisite geriatrics clerkship for fourth-year medical students: a successful model for teaching the Association of American Medical Colleges core competencies. Jornaul American Geriatric Society. 2009;57(10):1917-1924.

32. Gill TM. Geriatric medicine: it's more than caring for old people. American Journal of Medicine. 2002; 113(1): 85-90.

33. Eleazer GP, Doshi R, Weiland D et al. Geriatric content in medical school curricula. Results of a national survey. J Am Geriatr Soc 2005;53:136-140.

34. Ruiz JG, Teasdale TA, Hajjar I, Shaughnessy M,. Mintzer MJ. The Consortium of E-Learning in. Geriatrics Instruction. J Am Geriat Soc. 2007; 55:458-463.

35. Brasil. Poder Executivo, Decreto 1.498, de 3 de julho de 1996. Regulamenta a Lei $n^{\circ} 8842$ de 4 de janeiro de 1994 
que dispõe sobre a Política Nacional do Idoso, e dá outras providências. Diário Oficial [da] República Federativa do Brasil, Brasília, DF, 4 jul. 1996. Seção 1, ano 134, n. 128, 12278-12279.

36. Brasil. Ministério da Saúde. Política Nacional de Saúde do Idoso. Portaria n. ․ 1.395, de 9 de dezembro de 1999. Diário Oficial [da] República Federativa do Brasil. Poder Executivo, Brasília, DF, 13 dez 1999. N. 237-E, seção 1, p. 20-24.

37. Brasil. Lei n.8.080 de 19 de setembro de 1990 e Lei 8082 de 1992. Dispõem sobre as condições para a promoção, proteção e recuperação da saúde, a organização e o funcionamento dos serviços correspondentes e dá outras providências. Diário Oficial da União, Brasília, 1990. Seção1, p.18055 - 18059 .

38. Brasil. Diário Oficial da União. Lei no. 10.741, de 01 de outubro de 2003. Dispõe sobre o Estatuto do Idoso e dá outras providencias. Brasília; 2003.

39. Brasil. Ministério da Saúde. Portaria no. 2528, de 19 de outubro de 2006. Aprova a Política Nacional de Saúde da Pessoa Idosa. Brasília; 2006.

40. Diogo MJD'E. Formação de recursos humanos na área da saúde do idoso. Rev. Latino-Am. Enfermagem. 2004; 12(2): 280-282.

41. Association for Gerontology in Higher Education.[Internet]. Washington, DC 2014. [capturado 1 ago 2014]. Disponível: em http:/ /www.aghe.org/.

42. Europe Union Medicine Geriatric Society. Special Interest Group (SIG). [Internet].São Paulo;.2014. [capturado 20 jul 2014]. Disponível em http://www.eugms.org/index. php/meetingscourses/8-aboutus/interestgroups/401-education-and-training-in-geriatrics

43. Comissão Nacional de Residência Médica. [capturado 22 out 2006]. Disponível em: www.mec.gov.br/ensinosup/ hospital-universitário/residência/legislação.

44. Sociedade Brasileira de Geriatria e Gerontologia. [Internet]. Rio de Janeiro: SBGG; 2009. [capturado 13 mai 2014]. Disponível em: https:/ /www.sbgg.org.br/default.aspx.
45. Komatsu RS. Aprendizagem baseada em problemas: sensibilizando o olhar para o idoso. São Paulo: FAMEMA; 2003. p.13-25.

46. Motta LB. Formando médicos para o desafio do envelhecimento com qualidade no Brasil: uma contribuição à elaboração de currículos. Rio de Janeiro; 2004. Mestrado [Dissertação] - Universidade do Estado do Rio de Janeiro; Instituto de Medicina Social.

\section{CONTRIBUIÇÃO DOS AUTORES}

Autor Principal:

1.Concepção, delineamento do estudo;

2.Aquisição dos dados;

3.Interpretação dos dados;

7.Redação do manuscrito;

8.Revisão crítica.

Co- autor:

1.Concepção, delineamento do estudo;

2.Aquisição dos dados;

3.Interpretação dos dados;

5.Exames macroscópicos e histopatológicos;

7.Redação do manuscrito;

8.Revisão crítica.

\section{CONFLITO DE INTERESSES}

Declarou não haver.

\section{ENDEREÇO PARA CORRESPONDÊNCIA}

Vitor Jorge Woytuski Brasil

Rua Dom Pedro I, 342

Água Verde - Curitiba

CEP 80620-130 - PR

E-mail: vitorger@yahoo.com.br 